\title{
DE LA PÉdAgogie RELIGIEUSE DANS L'ÉLABORATION DE CAHIERS DE PRIÈRES VIA DES TUTORIELS SUR YOUTUBE
}

\author{
Kelber Pereira Gonçalves ${ }^{1}$
}

\begin{abstract}
Cette recherche s'efforce de mieux comprendre comment se constitue le processus d'une pédagogie religieuse au travers des productions audiovisuelles publiées et diffusées par de jeunes femmes laïques chrétiennes brésiliennes sur la plateforme YouTube. Ces productions, sous forme de « tutoriels », ont pour ambition d'apprendre aux autres internautes à élaborer un cahier de prières ou journal spirituel, et de les inciter à une pratique dite dévotionnelle visant en partie, dans la conception de ces internautes, à vivre une vie ascétique chrétienne.
\end{abstract}

\section{Introduction}

Avec le développement des nouvelles technologies de l'information et de la communication (NTIC) et notamment l'essor du modèle d'internet collaboratif ou " internet 2.0 », nous assistons, depuis quelques années, à une expansion incessante de la production, de l'appropriation, de la diffusion et réception de contenus religieux (Hoover $\&$ Clark, 2002). Dans le champ des religions, les nouvelles technolo-

1 Kelber Pereira Gonçalves est doctorant en Sciences de l'Information et de la Communication à l'Université de Tours et membre du laboratoire P r i m, Pratiques et ressources de l'information et des médiations.

Recherches en communication, $\mathrm{n}^{\circ} 53$ - Article publié le 15/09/2021 
gies ont contribué à une réorganisation de la religion dans la sphère sociale (Duteil-Ogata et al., 2015) et ont promu une reconfiguration de la manière dont les croyances et les pratiques religieuses sont pensées, transformant l'espace numérique en un « endroit » propice à la « création d'un environnement sacré » (Campbell, 2010, p. 9). Les nouvelles technologies et l'usage du numérique à des fins religieuses transforment non seulement l'expérience de la foi chez les croyants, mais aussi les religions en elles-mêmes (Hoover, 2006). Parmi les innombrables dispositifs numériques - blogs, sites, réseaux sociaux numériques, encyclopédies etc. - YouTube se présente comme une source féconde de transmission de contenus info-communicationnels concernant le salut, mais aussi comme " un lieu de circulation de savoirs et de savoirs d'action » (Douyère, 2019, p. 239). Sur cette plateforme, nous retrouvons des vidéos permettant aux internautes de regarder une messe en direct ou en différé, d'assister à des productions cinématographiques à contenu religieux, d'écouter des prédications, de la musique gospel, des louanges ou encore du chant grégorien. L'on peut également observer en direct l'image de la Vierge dans sa grotte à Lourdes ou réciter une neuvaine ${ }^{2}$ en ligne à Marie " qui défait les nœuds »- figure mariale qui, d'après la croyance catholique répandue notamment en Amérique latine, possède le pouvoir d'enlever les «nœuds » de la vie de croyants, c'est-à-dire de résoudre des problèmes compliqués - et même, grâce aux productions audiovisuelles du genre « tutoriel», apprendre à prier le Chapelet de la miséricorde divine ${ }^{3}$, ou encore apprendre à élaborer un cahier de prières.

Partant d'une première démarche qui se veut exploratoire et " compréhensive », cette recherche s'efforce de mieux comprendre comment se constitue le processus d'une pédagogie religieuse. Nous appréhendons la pédagogie comme « la transformation de la conscience qui a lieu à l'intersection de trois organes - l'enseignant, l'apprenant et les connaissances qu'ils produisent ensemble » (Lusted, 1986, p. 3)

2 Il s'agit dans la tradition chrétienne et catholique d'un exercice de piété, que le croyant répète pendant neuf jours consécutifs afin d'obtenir une grâce ou bien pour honorer un saint.

3 Il s'agit de la prière du chapelet adressée à Dieu le père par la miséricorde du Christ, tel que ce dernier lui-même l'aurait révélée de manière privée à sœur Faustine (Hélène Kowalska, sainte polonaise du $\mathrm{Xx}^{\mathrm{e}}$ siècle, 1905-1938). Bien que cette pratique de piété puisse être réalisée à tout moment de l'année, le début de la neuvaine est traditionnellement fixée le Vendredi saint, préférablement à 15 heures, heure à laquelle, dans la tradition chrétienne, le Christ aurait succombé à sa passion. 
à travers des productions audiovisuelles publiées et diffusées par de jeunes femmes laïques chrétiennes brésiliennes sur la plateforme YouTube. Il s'agit de productions sous forme de «tutoriels » ayant pour but d'apprendre aux autres internautes à élaborer un cahier de prières ou journal spirituel et de les inciter à une pratique dévotionnelle quotidienne ${ }^{4}$. Il nous importe ici de comprendre comment le cahier de prières est présenté par ces jeunes femmes sur YouTube en tant que méthode pédagogique d'organisation de prière et artefact central dans la pratique religieuse dite dévotionnelle.

Pour mieux comprendre le déroulement de la transmission des méthodes pour l'élaboration du cahier de prières lors de cette petite liturgie domestique, nous avons constitué un corpus de sept vidéos produites par des youtubeuses afin de saisir des similitudes et différences en ce qui concerne les éléments (décor, rythme, présence ou absence de son, scénario etc.) présents dans leurs productions audiovisuelles. Ce corpus, bien que limité, nous permettra de saisir d'une part les principes de cette pratique de piété populaire et d'autre part comment et par quelles méthodes, cette pratique est incitée à travers les tutoriels YouTube. Nous espérons, idéalement, être à même de susciter d'autres recherches ultérieures à propos de cette pratique encore peu étudiée. Le corpus s'est constitué à partir de l'insertion des mots clefs cadernos de oração (cahiers de prières) sur le moteur de recherche YouTube. À partir de la première vidéo proposée par YouTube, un ensemble de sept vidéos composant notre corpus s'est créé de manière automatique à partir de l'option « reproduction automatique » proposée par la plateforme. Bien que les questions techniques liées à cette fonctionnalité (notamment concernant les algorithmes ${ }^{5}$ ) mériteraient d'être étudiées, elles dépassent largement le cadre de cette étude. Étant donné qu'il n'y a pas à ce jour de consensus sur une seule méthodologie d'analyse de vidéos (Aumont \& Marie, 2015), nous procédons à une démarche qui se fera essentiellement en deux étapes : en premier lieu décomposer ou décrire, et en deuxième lieu tenter de saisir les relations entre les éléments décomposés sous la perspective d'une pédagogie religieuse, à savoir tenter d'interpréter ces productions (Vanoye, 2006). Il s'agit

4 Il s'agit, d'après ces internautes, du temps quotidien qu'elles consacrent à la communion avec Dieu. Cette communion se fait à partir de la prière, la lecture de la Bible et l'usage des cahiers de prières.

5 Voir à ce sujet: Cardon, D., À quoi rêvent les algorithmes? Nos vies à l'heure du Big Data, Paris, Seuil, 2015. 
de prendre en compte les aspects dits internes à la production audiovisuelle, à savoir les éléments esthétiques et langagiers présentés dans la vidéo, tout en prenant en compte les aspects dits externes à ces productions, liés aux temporalités correspondant à « la suite d'un ensemble de relations et les contraintes dans lesquelles se sont déroulées leur production et réalisation, tels que le contexte social, culturel, politique, économique, esthétique et technologique » (Penafria, 2009, p. 7). Nous proposons également une approche faisant appel à un cadre théorique interdisciplinaire qui conjugue principalement les études en communication et religion en contexte numérique (Douyère, 2014, 2018, 2019 ; Helland, 2005 ; Campbell, 2010), les concepts venant du champ de la théologie chrétienne (Sevez, 2018; Trottmann, 2003) et les sciences de l'éducation (Siqueira de Santana, 2011 ; Bonifácio \& de Freitas, 2011). Enfin, nous cherchons à démontrer que, quand bien même l'incitation à cette pratique pieuse à travers la présentation de méthodes pour l'élaboration du cahier de prières peut sembler nouvelle ou renouvelée - du moins, par son mode de transmission via une plateforme numérique -, elle s'inspire de pratiques bien plus anciennes que l'on pourrait croire. Ces pratiques trouvent leurs racines dans une tradition pédagogique chrétienne ancienne conjuguant la lecture des Écritures saintes, les méthodes de prière et les cahiers. Ce travail se présente en cinq parties. Dans une première partie, nous proposons de retracer de manière brève la tradition chrétienne de l'usage des cahiers ou guides de prière comme outils d'appui à la prière et par conséquent de communication avec le dieu chrétien. Dans une deuxième partie, nous tentons d'enquêter sur l'origine de l'usage des cahiers de prières en tant que pratique de piété en contexte brésilien. Dans un troisième temps, nous allons nous intéresser aux productions audiovisuelles des jeunes femmes laïques chrétiennes à proprement parler. Nous allons notamment nous intéresser à leur conception du cahier de prières, à l'usage qu'elles en font ainsi qu'aux motivations pour ces productions audiovisuelles. Dans une quatrième partie, nous examinerons les différentes méthodes pédagogiques proposées par ces jeunes femmes pour l'élaboration d'un cahier de prières. Enfin, dans une cinquième partie, nous nous intéresserons à l'interaction des internautes en analysant les commentaire laissés au sujet des vidéos publiées. Puisqu'il s'agit d'une pédagogie basée sur la théorie et les méthodes pour " faire faire » et inciter à une pratique, il s'agit dans cette partie du travail de tenter de saisir l'intérêt des internautes pour élaborer à leur tour un cahier de prières. 


\section{Lire, écrire et prier pour bien communiquer avec Dieu}

Nombreux sont les écrits pour penser la signification de la prière et pour en théoriser les méthodes : ils ont été élaborés dès les premiers siècles de la chrétienté dans la littérature patristique ${ }^{6}$. Dans $D e ~ l a$ prière, Origène (vers 184-254), le père de l'exégèse biblique affirmait que « celui qui prie comme il faut et selon ses moyens retire de multiples avantages de la prière » (Hamman, 1952, p. 397). Pour Origène, les trois sens de la lecture qui devraient être réunis lors de la lecture des textes sacrés, sont les sens littéral, moral et mystique, correspondant au trinôme " corps, âme et esprit ». La pensée d'Origène à propos du caractère indissociable de la lecture des Saintes Écritures et de la prière a été repris des décennies plus tard par Jérôme de Stridon (347-420), luimême lecteur et admirateur des écrits d'Origène (Jay, 1980). La pensée d'Origène concernant les lectures des textes sacrés a été renouvelée par Jean Cassien (360-435) et a nourri toute la vie monastique médiévale (de Lubac, cité dans Roques, 1960). Dans Traité de la prière, véritable manuel ou guide de prières, Évagre le Pontique (345-399) expose ses 153 maximes pour la prière chrétienne et les conditions endogènes et exogènes pour faciliter cette pratique qui est, d'après lui, un « entretien de l'intelligence avec Dieu » (Horvath, 2019, p. 1). Les entrelacs entre lecture des Saintes Écritures, écriture et prière demeurent également le thème central dans Confessions, œuvre majeure écrite à la fin $\mathrm{du} \mathrm{III}^{\mathrm{e}}$ siècle par Augustin d'Hippone (354-430). Largement étudié au fil des siècles, le texte peut être appréhendé à la fois en tant que journal intime ou journal spirituel et manuel pédagogique. Le texte des Confessions ne comporte pas seulement la confession des péchés d'Augustin d'Hippone, mais aussi un « témoignage» de vie ascétique chrétienne au service d'une " pédagogie de transmission de la foi » (Pierron, 1995, p. 265). Quelques siècles plus tard, Guigues Ir le Chartreux (1083-1136) et Guigues II le Chartreux (1114 - vers 1193), respectivement cinquième et neuvième prieur du monastère de La Grande Chartreuse, ont contribué, en contexte français et européen, à la diffusion de la conception de la prière en tant qu'exercice de méditation et de contemplation intersecté à la lecture des textes sacrés. Guigues ${ }^{\text {er }}$ le Chartreux, dans Coutumes de Chartreuse, qualifie la prière comme un exercice central de la vie monastique : « [...] La douceur de la psalmodie, l'application à la lec-

6 Il s'agit des textes écrits par les «Pères de l’Église », les théologiens et auteurs chrétiens des premiers siècles après Jésus-Christ. 
ture, la ferveur de la prière, la profondeur de la méditation, le ravissement de la contemplation » (Guigues le Chartreux cité dans Becdelièvre, 2003, p. 219). Guigues exprime le caractère insécable entre lecture et prière de la manière suivante : " cherchez en lisant, et vous trouverez en méditant ; frappez en priant, et il vous sera ouvert par la contemplation » (Catéchisme de l'Église catholique, $\mathrm{n}^{\circ}$ 2654). La contribution de Guigues II dans sa Lettre de la vie contemplative, encore appelée Échelle des moines, écrite à la fin du $\mathrm{XII}^{\mathrm{e}}$ siècle, a consisté en la reprise et la propagation des quatre étapes de la vie contemplative de la lectio divina - notion qui trouve ses racines dans la pensée d'Origène. La notion de lectio divina, attribué à Cyprien de Carthage (vers 200-258), largement employée dans tout le Moyen Âge consiste à établir un dialogue avec Dieu à travers la lecture et les prières : « un dialogue intime avec un interlocuteur divin vivant et présent qui répondra lorsque le lecteur l'appellera » (Robertson, 2011, p. 12). Quatre piliers fondent cette communication avec Dieu, à savoir : lecture, méditation, prière et contemplation :

« La lecture est l'étude attentive des Écritures faite par un esprit appliqué. La méditation est une opération de l'intelligence, procédant à l'investigation studieuse d'une vérité cachée, à l'aide de la propre raison. La prière est une religieuse application du cœur à Dieu pour éloigner des maux ou obtenir des biens. La contemplation est une certaine élévation en Dieu de l'âme attirée au-dessus d'elle-même et savourant les joies de la douceur éternelle » (Trottmann, 2003, p. 265).

Cette méthode - conjointement à l'obéissance, l'humilité, le travail et la liturgie monastique - employée pendant tout le Moyen Âge, a traversé les siècles et subsiste encore de nos jours, pratiquée par les bénédictins, les cisterciens, les ignaciens, les carmes et carmélites ou encore, par des courants du renouveau charismatique catholique. Contemporain à Guigues II, le fondateur de l'ordre des prêcheurs, Dominique de Guzmán (1170-1221) quant à lui, pratiquait un mode de prière ayant recours aux cinq sens du corps (Palazzo, 2016, 2017). Cette méthode de prière a été transcrite après la mort du religieux par les dominicains sur un manuscrit intitulé Neuf modes de prière de saint Dominique. Cette méthode de prière fondée sur des mouvements tels que prosternation, génuflexion, cris, pleurs, gémissements, prières 
à mains ouvertes ou bras tendus, visait à communiquer la prière par l'image du corps (Douyère, 2014) et a considérablement contribué à définir et consolider la spiritualité de l'Ordre.

Thomas d'Aquin (1225-1274), pour sa part, mène aussi dans sa Somme théologique (première publication en latin en 1485) un certain nombre ${ }^{7}$ de réflexions à la fois théologiques et philosophiques sur l'importance et le « sens » de la prière dans la vie du chrétien. Il ne s'agit pas, dans l'ouvrage, d'élaborer des formes de prières censées être imitées, mais plutôt d'énumérer des questions à propos de la prière présentées sur le modèle de questions-réponses structurées. Au cours de la Renaissance, l'ouvrage Exercices spirituels (première publication en latin en 1548), élaboré par Ignace de Loyola (1491-1556), fondateur de la Compagnie de Jésus, entretient la tradition chrétienne d'investissement dans l'élaboration des guides pédagogiques de prière. L'ouvrage, fortement inspiré par Ludolphe le Chartreux (vers 1300 - vers 1378), reprend et diffuse la notion de "discernement » élaborée par Paul de Tarse et méditée par les Pères de l'Église (Hernández, 2020) et propose une systématisation des modes de prière. Il s'agit d'un ouvrage qui peut être appréhendé comme " objet culturel capable de préfigurer des pratiques et des modalités de construction d'une forme de vie spirituelle » (Catellani, 2016, p. 85). Dans ce guide ou manuel de prières, Ignace met en exergue les dimensions communicationnelle et pédagogique de la prière qui ne doit pas seulement être pratiquée, mais aussi enseignée ou transmise. Ignace parle de « pédagogie de l'intelligence » qui se constitue à partir d'un « vase incessamment communicant entre vie intellectuelle et expérience spirituelle» (Sevez, 2018, p. 79). La notion d'imagination tient également une place privilégiée dans Exercices spirituels. Loyola invite le lecteur à méditer sur les œuvres des arts sacrés, faisant recours à un " imaginaire » ayant pour but de " faire passer d'une piété formelle à l'expérience d'une foi vécue, le transportant sur un mode affectif et visionnaire à l'intérieur même de son sujet de méditation »(Greiner, 2012, p. 98). Ce n'est pas par hasard que l'usage de l'image - mais aussi le théâtre -, si cher à Ignace de Loyola sera essentiel comme outil pédagogique dans la transmission de la doctrine et de la foi catholique par des missionnaires jésuites durant le processus de catéchisation des peuples, notamment ceux dont la langue faisait barrière.

7 Secunda secundae, question 83, La prière, articles 1-17. 
Or, si cette pratique est ancienne dans ce que l'on pourrait appeler une tradition chrétienne et catholique, qu'en est-il de l'usage des cahiers de prières dans la contemporanéité en contexte brésilien ? Quelle serait l'origine de l'usage de cette pratique de piété dans le pays ? Enfin, comment l'incitation à une pratique pieuse si ancienne, associant la lecture, l'écriture, la prière et la médiation se renouvelle-t-elle, du moins dans sa forme, dans l'environnement numérique à travers des tutoriels d'élaboration de cahiers de prières sur la plateforme YouTube?

\section{Les cahiers de prières : une pratique pieuse « populaire » encore peu étudiée}

$\mathrm{Au}$ moment même où nous écrivons ces lignes, la version brésilienne du moteur de recherche Google (google.com.br) présente environ 9.900.000 résultats pour caderno de oração (en portugais et au singulier) et 4.390.000 résultats pour cadernos de oração (en portugais et au pluriel). Parmi ces résultats, nous trouvons des contenus audiovisuels, des images, des textes et des cahiers de prières pré-remplis ou vierges disponibles dans des boutiques de vente en ligne. Dans l'affichage des « recherches associées » à caderno de oração, le moteur de recherche Google énumère, entre autres, « cahier de prière PDF gratuit », « cahier de prière décoré », " cahier de prière à acheter », " texte pour cahier de prière » ou encore « cahier de prière pour enfant ». En bref, si nous pouvons constater que de nombreux ouvrages religieux chrétiens portant sur cette pratique de piété sont disponibles sur internet, il n'en est pas de même en ce qui concerne les articles académiques. En raison de la carence d'études portant sur le sujet, une évaluation effective sur l'ampleur du phénomène au Brésil demeure à ce jour incertaine. Nous pourrions émettre l'hypothèse selon laquelle le manque d'intérêt scientifique pour les cahiers de prière au Brésil tiendrait, du moins en partie, au classement de cette pratique dans la catégorie de la piété populaire, c'est-à-dire des pratiques non inscrites parmi les pratiques canoniques.

En effet et d'après nos recherches, non seulement l'ampleur du phénomène mais aussi l'origine même de l'élaboration des cahiers de prières restent délicates à mesurer en tant que pratique de piété au Brésil. Nonobstant, quelques travaux en sciences de l'éducation nous apportent des pistes concernant les origines plausibles de cette pratique au Brésil. Des recherches menées par Siqueira de Santana sur les pratiques éducationnelles auprès d'un orphelinat catholique pour les jeunes filles dans le Nord-ouest brésilien ont démontré que l'usage des cahiers 
de prières était une pratique courante entre 1922 et 1969 - période correspondant à l'étude des archives de l'institution par la chercheuse. L'auteure constate que l'usage de cahiers de prières conjointement à la broderie, aux « cours des bonnes manières » et à l'enseignement religieux (programmes d'oraisons et participation quotidienne à la messe) étaient les ingrédients nécessaires pour assurer une formation convenable pour les jeunes filles défavorisées selon les préceptes de l'époque. Dans ce contexte et d'après ce modèle institutionnel, il était préférable « d'éduquer » plutôt que " d'instruire » afin de prévenir l'oisiveté, la prostitution et la mendicité et « de leur donner les conditions nécessaires pour s'insérer dans la société de leur temps et remplir un rôle social satisfaisant, c'est-à-dire, de bonnes mères et de femmes au foyer exemplaires » (Siqueira de Santana, 2011, p. 23).

D'autres travaux semblables, toujours en sciences de l'éducation, confirment le lien entre éducation religieuse, écriture et prières. Bonifácio et de Freitas ont mis en exergue l'inspiration de la pédagogie salésienne dans l'enseignement de la morale et de la religion dans une institution religieuse accueillant des mineurs démunis, orphelins ou abandonnés. La recherche met l'accent sur l'éducation formelle, c'est-à-dire conforme à celle déterminée par le ministère de l'Éducation brésilien, mais aussi informelle, à savoir celle découlant de l'ensemble des activités manuelles réalisées dans l'institution - « couture, broderie, confection des fleurs artificielles, tapisserie, techniques de dessin et de peinture, cours de poésie ou pour apprendre à bien s'occuper de la maison : cuisiner, laver, repasser, faire le ménage » (Bonifácio \& de Freitas, 2011, p. 632). Des entretiens menés avec d'anciennes élèves ont permis de mieux comprendre la vie quotidienne de ces jeunes filles aux activités religieuses et manuelles intenses. D'après le modèle pédagogique salésien, il est fondamental d'occuper le temps des élèves de manière dense - éducation formelle institutionnalisée, éducation morale, civique et religieuse et travaux manuels - afin d'éloigner ces jeunes filles « des vices déformateurs de l'oisiveté » (Bonifácio \& de Freitas, 2011, p. 613). Les cahiers de prières en ce contexte pourraient être pensés non seulement comme un journal intime et outil de « communication » avec Dieu, mais aussi comme un outil d'incitation à une pratique relevant du travail manuel dans l'objectif peut-être à la fois de combler des moments d'oisiveté et de renforcer l'appartenance confessionnelle et communautaire, tout en développant leur habileté manuelle et leur créativité artistique. Enfin, dès lors que l'incitation à l'élaboration et à l'usage des cahiers de prières se destine aux jeunes femmes, 
nous pouvons supposer que cette pratique de piété est à même de fonctionner aussi comme ustensile supplémentaire de délimitation des rôles de genre.

Enfin, la catégorisation en tant que pratique religieuse « populaire » de cette pratique de piété peut aussi être entendue comme exprimant une opposition à une autre forme de religion qui serait entendue comme « érudite » - le terme cacherait ainsi des dynamiques de pouvoir (voire même de domination) entre ces deux pôles. Sous une approche socio-économique, le terme « populaire » comporte une idée secondaire qui s'y rattache : il peut connoter une condition socio-économique des individus qui appartiendraient aux classes sociales les moins aisées. Au-delà du caractère populaire de l'usage des cahiers de prières, le manque d'intérêt scientifique pour cette activité pourrait dériver, sous une perspective foucaultienne, de l'espace où celle-ci était (ou l'est encore ?) pratiquée (à savoir l'orphelinat en tant qu'institution de renfermement) ainsi que des individus qui la pratiquaient (ou la pratiquent), à savoir, des enfants pauvres orphelins ou abandonnés.

\section{3. Élaborer un cahier de prières sur YouTube : quels acteurs?}

N'ayant pas accès aux données relatives au profil sociologique des acteurs étudiés, nous avons choisi, dans le cadre de cette étude, d'utiliser le terme « jeunes femmes », car suite au visionnage des productions audiovisuelles, nous supposons qu'il s'agit d'internautes ayant entre 15 et 35 ans environ. En ce qui concerne la filiation religieuse des vidéastes étudiées, à l'exception de Sarah Néry qui précise faire partie de la communauté charismatique catholique Shallon ${ }^{8}$, les autres jeunes femmes ne déclarent pas leur(s) appartenance(s) confessionnelle(s) - le phénomène des multi-appartenances n'est pas rare au Brésil - dans les vidéos analysées. Par leur vocabulaire (elles parlent de culte et non de messe par exemple), nous supposons qu'il s'agit de femmes évangéliques ${ }^{9}$.

8 Il s'agit d'une communauté charismatique catholique créée dans le nord-est du Brésil dans les années 1980 .

9 En contexte brésilien, en raison de la multitude de confessions, nous considérons « évangélique » tout fidèle chrétien ne se déclarant pas catholique romain ni chrétien orthodoxe. Néanmoins, il est important de souligner que la catégorie « évangélique » demeure très hétérogène, et a une signification différente dans d'autres parties du monde. 
D'ailleurs, dans les commentaires laissés par d'autres internautes sur la vidéo de Sarah Néry, nous pouvons constater des messages d'autres jeunes femmes se déclarant catholiques, mais aussi évangéliques, tenant à féliciter les vidéastes pour leur tutorie ${ }^{10}$.

D'après la youtubeuse Francine Veríssimo, le cahier de prières peut être défini comme un journal intime ou journal spirituel, compris comme outil pédagogique de piété qui serait à même de rapprocher davantage les croyants de Dieu : «Premièrement, le journal spirituel t'aide à organiser et donner de la clarté à tes pensées. L'écriture a ce pouvoir de nous aider à catégoriser nos pensées [00':22"-00':48”]. Deuxièmement, le journal spirituel t'aide à apprendre davantage sur la parole de Dieu. Par-dessus tout, le journal spirituel est un outil d'apprentissage » (00':53"-00':57”). Elle souligne que le cahier de prières peut fonctionner comme outil de communication pour « discuter avec Dieu » et mieux comprendre ce que Dieu a à leur dire, car c'est grâce au cahier de prières que : « nous allons pouvoir écrire les versets qui nous ont sauté aux yeux tel jour, ce que nous sommes en train d'apprendre à l'Église, les messages, ou ce que le Seigneur, à travers le SaintEsprit dit à notre cœur. C'est une espèce de cahier dans notre école de la grâce. C'est avec lui que nous allons grandir en parfaite connaissance de notre Seigneur et sauveur Jésus-Christ » (00':50"-01':23”). L'usage du cahier de prières s'inscrit au cœur de la pratique dévotionnelle quotidienne cultivée par ces jeunes femmes. Cette pratique dévotionnelle, nous explique Michelle Souza, consiste à « réserver une période significative de la journée pour être en communion avec Dieu »; [Il s'agit de] « prendre du temps pour discuter avec lui, pour lire sa parole, l'étudier, c'est-à-dire réserver du temps pour adorer Dieu » (00':32"00 ':49"). Concernant la durée recommandée, il s'agit d'après Michelle, de prendre « au moins une petite heure de la journée pour la consacrer à Dieu, pour garder du temps pour étudier ses écritures, pour garder du temps pour discuter [...] avec lui » (00':50"-01':21"). Enfin, le cahier de prières peut contenir des registres écrits d'oraisons ou prières, des transcriptions de versets bibliques, paraboles, cantiques ou psaumes, ainsi que des annotations concernant, entre autres, des remerciements pour les grâces obtenues. Pour ce qui est de la pratique dévotionnelle, Michelle Souza explique qu'il est important de « commencer par deman-

10 Par exemple, nous pouvons lire dans le commentaire de l'internaute Izabella Borges : «Merci, sœur catholique. Que nos religions ne soient pas des barrières au partage de notre amour de Dieu et des enseignements chrétiens ». 
der au Saint-Esprit de nous guider dans les écritures » (4':33"-4':36"). Puis, il est question de choisir un horaire, et faire de cet horaire une habitude, ainsi qu'un endroit tranquille, " un petit coin qui ne soit qu'à toi » (2':20"-2':23"). Toujours d'après Michelle Souza, " La parole de Dieu est plus actuelle que le journal de demain » (7':30" -7':34") c'est pourquoi elle souligne qu'il est important de lire et relire la Bible : " demande au Saint-Esprit, lis la Bible avec calme, virgule par virgule, calmement. Ne sois pas pressé. La Bible doit être examinée » (6':50"7':10"). C'est pourquoi, toujours d'après elle, il est important d'élaborer un plan de lecture ou de préférence se procurer dans des librairies spécialisées des livres dédiés à la pratique dévotionnelle pour s'initier » (3':50" jusqu'à 4':00”).

\section{Le cahier de prières : composition aux méthodes multiples}

Dans le cadre de la pratique dévotionnelle quotidienne, l'une des méthodologies suggérées par la vidéaste Tainá Brito - qui rappelle les principes de la lectio divina - est celle du " $\mathrm{SOAP}^{11} »$ (acronyme de scripture, observation, application, prayer). L'écriture s'inscrit dans la rédaction et la retranscription de remarques ou de notes à propos des chapitres ou versets bibliques préalablement lus. L'observation relève du résumé des textes et de la méditation sur « ce que Dieu a voulu dire » $\left(01^{\prime}: 46 "-01^{\prime}: 48\right.$ "). L'application consiste à employer la vérité apprise de manière pratique dans la vie du croyant. Enfin, prayer correspond à la prière établie sur ce qui a été appris. Pour son cahier de prières, Sarah Néry explique avoir ajouté six catégories en plus de la catégorie " prière ", à savoir groupe de prière, confession, acomp's (accompagnement), dates importantes, surprises de Dieu et « discernement »notion visiblement inspirée de la théologie ignacienne. Elle explique encore qu'elle utilise d'autres livres chrétiens en complément de sa pratique dévotionnelle quotidienne. Les abréviations EB (étude biblique)

11 Si nous n'avons pas été à même de déterminer l'origine de cette méthode, nous trouvons à partir d'une simple recherche sur le moteur de recherche Google un certain nombre de sites chrétiens qui expliquent le concept et l'utilisation de cette méthode. À titre d'exemple, d'après le site chrétien Christ Community Ministries la méthode SOAP « est une méthode intentionnelle et stratégique de lecture de la Bible et de journalisation. Intentionnelle car cela nécessitera un engagement. Stratégique car elle fournit un système qui vous permettra d'évaluer les progrès ». Disponible ici : http://www.ccmchurch.com/connect/s-o-a-p/ (dernière consultation le 17/12/20). 
et OP (oraison personnelle) sont également utilisées pour différencier ce qui relève des pratiques religieuses collectives dans le cadre de son groupe charismatique et celles concernant sa pratique pieuse personnelle. Enfin, dans les sections telles que confession, le lieu visité à cet effet, la date, l'adresse, la pénitence et les conseils donnés par le prêtre sont également enregistrés dans le cahier de Sarah Néry.

Fabiola Keffer, quant à elle, explique que lors de ses lectures bibliques, elle utilise une forme de méditation ( $7^{\prime}: 40^{\prime \prime}-77^{\prime}: 50$ ") consistant à " s'imaginer dans l'histoire ", ce qui renvoie une fois de plus à la tradition ignacienne relevant de la " vision imaginaire " à partir des lectures des Saintes Écritures. De l'ascétisme chrétien en contexte numérique est également présent dans le modus operandi de la méthodologie de la prière développée par l'une de ces vidéastes, à partir des dynamiques de renoncement et de (re)négociations courants en contexte de pratique religieuse numérique (Campbell, 2010). En ce sens, Tainá Brito explique que la devise mise en pratique dans sa vie [elle fait signe avec les mains et demande l'inclusion du 'hashtag'] \#SemDevocionalSemRedeSocial (pas de dévotionnel, pas de réseaux sociaux) repris, d'après elle, à partir de la lecture « du blog de Deby » (0':20"-0':40"), dont nous supposons qu'il s'agit d'un blog à contenu religieux. Ainsi Tainá Brito cherche à vivre une ascèse chrétienne non seulement en dehors de l'environnement numérique, mais aussi en contexte numérique (Jonveaux, 2007). Cela confirme une fois de plus le constat selon lequel l'expérience du religieux dépasse la simple possibilité de cliquer sur des hyperliens et de recevoir des informations (Helland, 2005, p. 12) et s'inscrit dans des dynamiques complexes relevant d'un grand nombre de subjectivations religieuses caractéristiques de la modernité religieuse (Hervieu-Léger, 1987). Sarah Néry, de son côté, utilise deux cahiers de prières : un premier est, d'après elle, « bien plus petit et super mignon, bien plus spiritualisé » et un deuxième, plus « rationnel » affichant en couverture la représentation du personnage de bande dessinée The Amazing Spider Man (8':00"-8':30"). Bien qu'elle ne développe pas davantage les définitions des termes « rationnel » et «spiritualisé », les deux cahiers de prière ont donc des fonctions différentes : le premier fait office d'agenda afin de documenter des pratiques religieuses institutionnalisées et collectives et l'autre est dédié à la pratique dévotionnelle individuelle. Tainá Brito, pour sa part, possède également deux cahiers de prières : l'un destiné exclusivement à l'étude des Saintes Écritures et l'autre pour « les prières à proprement parler »-elle ne développe pas davantage son propos. 


\section{Des tutoriels pour faire faire : « je vais faire un cahier de prières tout de suite ! "}

Apprendre aux internautes à élaborer un cahier de prières à partir de ces productions audiovisuelles - comme le genre audiovisuel « tutoriel » même le suggère d'emblée - va de pair avec la pédagogie telle qu'elle a été pensée par Durkheim, à savoir celle qui « consiste, non en actions, mais en théories. Ces théories sont des manières de concevoir l'éducation, non des manières de la pratiquer » (Durkheim, 1922, p. 75). Apprendre aux internautes à élaborer un cahier de prières trouve un écho dans le but même de la pédagogie, si nous avons toujours recours à la pensée durkheimienne, à savoir non pas de "se substituer à la pratique, mais de la guider, de l'éclairer, de l'aider, au besoin, à combler les lacunes qui viennent à s'y produire » (Durkheim, 1922, pp. 95-96). Les productions audiovisuelles étudiées guident ces internautes désireuses d'élaborer un cahier de prières. Si nous partons du présupposé selon lequel « la communication comme production de sens fondamentalement altérisée, engage l'autre et peut faire faire » (Douyère, 2018, p. 223), l'action d'incitation à une pratique pourrait elle-même être comprise comme une pratique : faire faire (un cahier de prières) à partir des billets des tutoriels YouTube. Dans cette transmission de savoirs, des méthodes concrètes sont proposées aux internautes afin de parvenir avec succès à la pratique dévotionnelle - et, par conséquent, bien « communiquer » avec le dieu chrétien - et confectionner un cahier de prières. Ainsi, pour ce qui est de la méthodologie de lecture des Saintes Écritures, Tainá Brito explique que sa Bible est « un petit peu colorée » (4':50"-4':58") puisqu'elle souligne avec des crayons de couleur les versets et les chapitres qui ont attiré le plus son attention. Cela parce que « le stabilo est trop fort et la page de la Bible est toute fine, donc les crayons de couleur ne tachent pas l'autre côté [le verso], ça c'est le meilleur côté » $\left(5^{\prime}: 10^{\prime \prime}-5^{\prime}: 25^{\prime \prime}\right)$, dit-elle. 


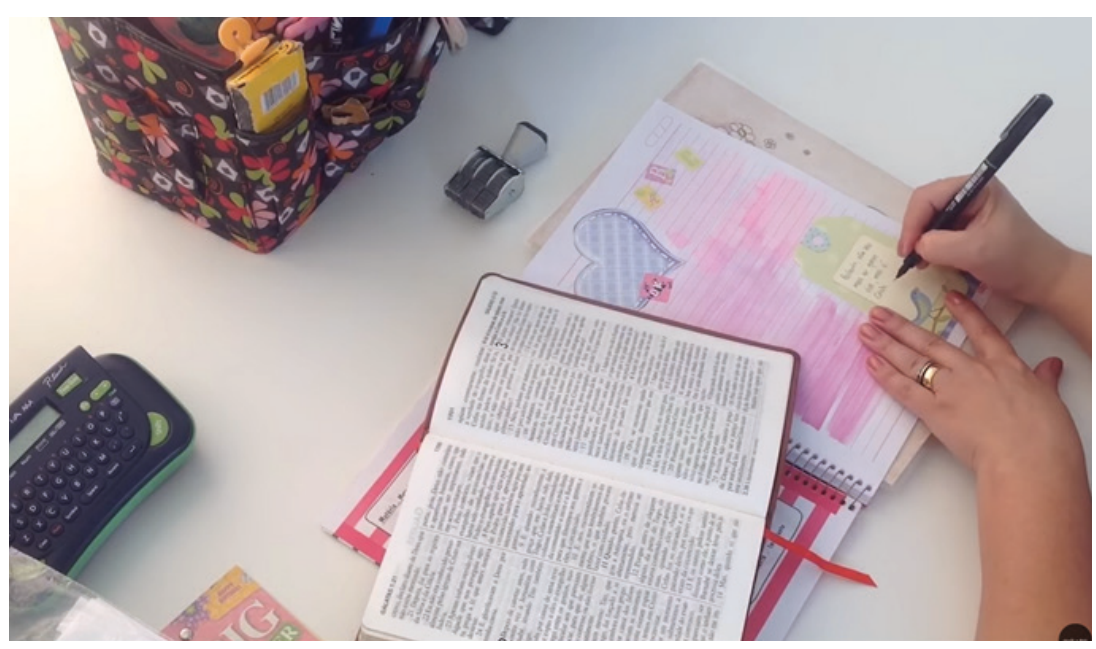

Figure 1. Capture d'écran de la vidéo intitulée "Devocional com Arte 5 Como eu uso o meu caderno de devocionais » de la vidéaste Duda Ruiz

De la même manière, dans sa vidéo intitulée Vocacional com Arte (dévotionnelle avec l'Art), la vidéaste Duda Ruiz indique les matériaux utilisés dans sa vidéo, information qui apparaît à la fin de la production audiovisuelle : les références des stylos utilisés, qualité ou marque du papier etc. Les précisions concernant les matériels utilisés peuvent se justifier par le souci esthétique qui tient également une place importante dans le cahier de prières : «la commande religieuse, le don pieux, la prière ne sont pas incompatibles avec la recherche de la qualité artistique et avec le plaisir esthétique » (Christin, 2002, p. 90). Ainsi, ces informations pratiques diffusées par Duda Ruiz peuvent être utiles aux internautes désireuses d'élaborer leurs cahiers de prières, l'utilisation des différentes couleurs de stylo, adhésifs, trombones, ficelles, marquepages, gouache, ou de techniques variées en collage ayant vocation à rendre « beau » le cahier de prières.

En dépit de leur désir d'élaboration d'un cahier de prières, certaines internautes semblent ignorer non seulement les méthodes pour $\mathrm{y}$ parvenir, mais l'existence même de cette pratique pieuse. Les très nombreux commentaires laissés par les internautes à propos de ces productions audiovisuelles - publiés dans leur totalité ou presque par des femmes - attestent d'un intérêt pour la confection des cahiers de prières et pour les méthodes pour y parvenir. Prenons comme exemple les commentaires laissés sous la vidéo de Sarah Néry. L'internaute Cris Moia écrit : « J'ai adoré ces astuces ! J'ai déjà vu des cahiers d'organi- 


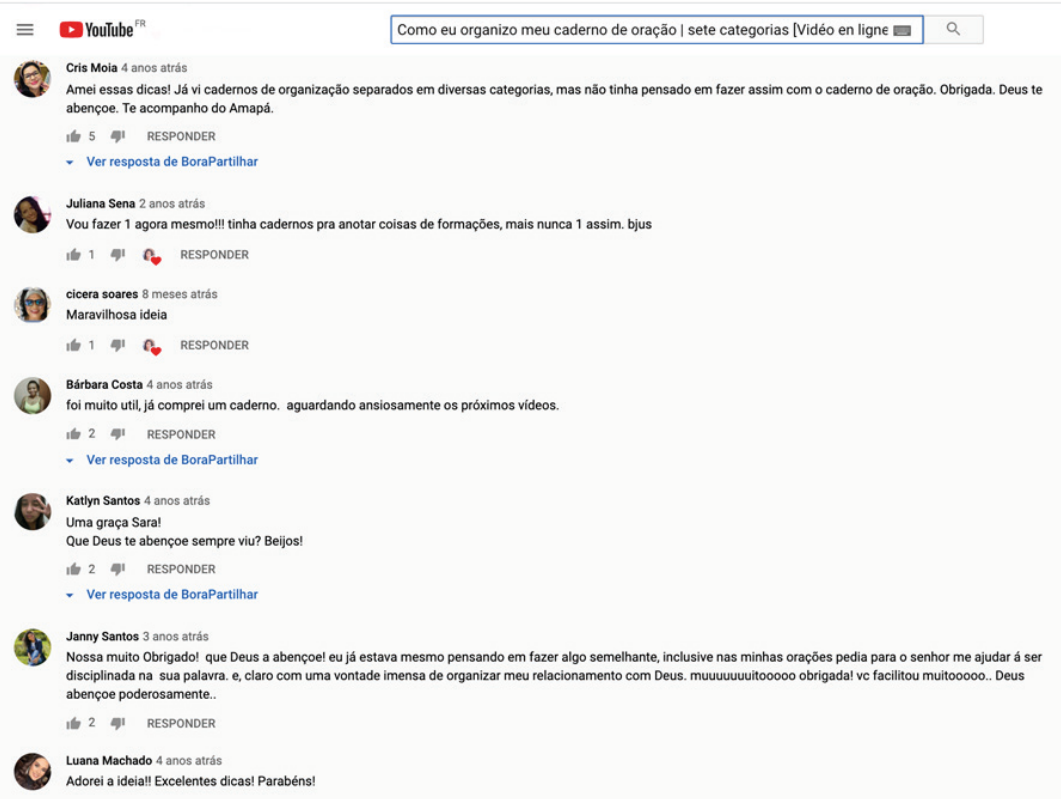

Figure 2. Capture d'écran de la vidéo intitulée "Como eu organizo meu caderno de oração - sete categorias » de la vidéaste Sarah Néry

sation divisés en diverses catégories, mais je n'avais pas pensé à faire comme ça pour le cahier de prières ». Juliana Sena fait aussi savoir : « Je vais faire un [cahier de prières] tout de suite ! J'avais des cahiers pour noter des choses de formation, mais jamais un comme ça ». Bárbara Costa pour sa part écrit : « [ça] été très utile. J'ai déjà acheté un cahier. J'attends avec impatience les prochaines vidéos ». Janny Santos quant à elle révèle : «Oh là là, merci beaucoup! Que Dieu te bénisse. J'avais pensé déjà à faire quelque chose de semblable, d'ailleurs dans mes prières je demandais au Seigneur de m'aider à être disciplinée dans sa parole et bien entendu avec une immense volonté d'organiser ma relation avec Dieu. Merci beaucoup. Tu [m'as] beaucoup aidée. [Que Dieu] te bénisse puissamment ! ». Les commentaires laissés par les internautes confirment ainsi l'existence d'une relation entre le vidéaste et les internautes : « la consultation de vidéos diffusées sur les chaînes YouTube personnelles participe donc d'une co-construction de la relation, figurée par le ou la vidéaste, mais aussi d'une croyance dans la présence ainsi figurée et partagée, et d'une co-construction imaginaire de la relation » (Douyère, 2019, p. 222). L'internaute Luana Machado se réjouit : « j’ai adoré l'idée ! Excellentes astuces ! Félicitations !». 
Ainsi, dans le cas de ces vidéastes, il ne s'agit pas véritablement de la médiatisation d'une pratique religieuse, mais plutôt d'une incitation à la pratique qui se fait par le biais d'une pédagogie religieuse ancrée dans la proposition de méthodes précises - qui peuvent être ou non plus ou moins imitées - servant de source d'inspiration aux internautes désireuses d'avoir leur propre cahier de prières.

\section{Conclusion}

Nous avons proposé une recherche exploratoire ayant pour but de mieux comprendre le processus d'une pédagogie religieuse à travers les productions audiovisuelles sur YouTube. Dans les tutoriels produits par ces jeunes femmes, la pédagogie religieuse s'inscrit dans l'incitation à la pratique et la mise en scène des méthodologies, des techniques, procédés et méthodes qui seraient susceptibles d'être employés par les internautes désireuses d'élaborer leur propre cahier de prières. Il ne s'agit pas de médiatisation en ligne d'une pratique pieuse à proprement parler - si l'on appréhende la médiatisation en tant que "modalités de présence des pratiques religieuses dans l'environnement médiatique » (Martino, 2017 : 100) -, mais d'incitation à une pratique. Cette incitation se justifie, d'après ces vidéastes, par un « vécu » ou une communication avec Dieu, qui serait favorisée par l'usage des cahiers de prières. Nous l'avons vu, la conjonction entre lecture, écriture et prières ainsi que la transmission de méthodes de prière à travers des manuels ou guides de prières demeurent anciennes dans l'histoire de la chrétienté. En contexte brésilien, et d'après nos recherches, l'usage du cahier de prières était courant dans certains orphelinats au Brésil afin de combler les moments d'oisiveté, de développer l'habileté manuelle et la créativité artistique et, probablement, comme outil supplémentaire de délimitation des rôles de genre. Car, si nous ne pouvons pas affirmer qu'il s'agit d'une pratique exclusivement féminine, la pratique du cahier de prières chez les jeunes garçons, d'après nos recherches, demeure inhabituelle au Brésil. En effet, à partir de l'insertion des mots clefs cadernos de oração - homem (cahiers de prières - homme) cadernos de oração masculino (cahiers de prières - masculin) sur le moteur de recherche de la plateforme YouTube nous ne trouvons pas de résultats portant sur des tutoriels réalisés par des garçons ou pour apprendre aux garçons à élaborer un cahier de prières. Lorsque nous procédons de la même manière et avec les mêmes mots clefs sur le moteur de recherche Google, nous retrouvons peu de résultats dont deux à trois modèles de 
cahiers de prière en vente sous le label de la communauté du renouveau charismatique catholique Shallon (la même communauté dont la youtubeuse Sarah Néry est membre). Ces cahiers en vente, différemment de ceux réalisés par les jeunes femmes étudiées, présentent des couleurs sobres, la plupart du temps en tons de noir et blanc. Sur la couverture de l'un de ces cahiers nous pouvons lire Seja forte e corajoso ${ }^{12}$ (soyez fort et courageux). En ce sens, les pratiques pieuses peuvent être pensées comme des mediums de communication entre le fidèle et son dieu, mais elles sont aussi des pratiques sociales à part entière accomplissant des rôles bien précis. Si nous ne pouvons pas non plus affirmer que les anciennes élèves de ces institutions ont transmis la pratique de l'usage du cahier de prières à leurs filles, nous pouvons constater en revanche qu'il s'agit d'une pratique répandue en dehors des murs des orphelinats. Comme l'attestent les nombreux résultats sur le moteur de recherche Google, il s'agit donc d'une pratique encore courante en contexte brésilien. En outre, si les pratiques sociales sont directement liées aux pratiques médiatiques (Martino, 2017), l'existence de nombreuses vidéos pour apprendre aux internautes à élaborer un cahier de prières sur YouTube confirmerait l'usage des cahiers de prières comme une pratique pieuse toujours d'actualité. Écrire des prières sur papier et produire des tutoriels pour apprendre aux internautes à élaborer des cahiers de prières sont visiblement des pratiques complémentaires. Néanmoins, ce « cours vidéo » ne possède pas seulement un caractère pédagogique ou organisationnel de la prière : il s'agit également de la présentation d'un " style » de vie basé sur la pratique dévotionnelle et la communion avec Dieu. Dans le processus de production de ces vidéos, il y a également une dimension qui ne nous est pas accessible, celle de l'édition : ont-elles prié pour que le Saint-Esprit puisse leur donner le discernement pour choisir la musique la plus adéquate pour accompagner leurs productions ? Ou bien, ont-elles demandé l'inspiration au Saint-Esprit pour conserver certains morceaux d'enregistrement au détriment d'autres? Dans une perspective d'éventuelles recherches futures, il serait intéressant de mener des entretiens avec ces vidéastes afin de mieux comprendre également le processus d'édition de ces productions audiovisuelles.

12 Disponible ici : https://www.elo7.com.br/caderno-devocional-masculino/dp/E10C0E (dernière consultation le 17/12/20). 


\section{Références}

Aumont, J. \& Marie, M. (2015). L'Analyse des films (3e éd.). Paris : Armand Colin.

Bonifácio, N. S. \& de Freitas, A. G. (2011). A educação formal e informal do oratório festivo "São João Bosco" para meninas abandonadas. Revista de Ciências da Educação, XIII(24), 611-646. Disponible à : https://ri.ufs.br/bitstream/riufs/1060/1/ AEduca $\% \mathrm{C} 3 \% \mathrm{~A} 7 \% \mathrm{C} 3 \% \mathrm{~A} 3 \mathrm{oF}$ ormal.pdf

Campbell, H. (2010). When Religion Meets New Media. New York (NY): Routledge.

Cardon, D. (2015). À quoi rêvent les algorithmes? Nos vies à l'heure du Big Data. Paris : Seuil.

Catellani, A. (2016). Écrire et instruire la méditation et la contemplation chrétiennes : les Exercices spirituels d'Ignace de Loyola. Communication \& langages, 3, 85-105. Doi : https://doi.org/10.4074/S0336150016013053

Christin, O. (2002). Du culte chrétien au culte de l'art : la transformation du statut de l'image ( $\mathrm{xV}^{\mathrm{e}}-\mathrm{XVIII}{ }^{\mathrm{e}}$ siècles). Revue d'histoire moderne \& contemporaine, 49-3(3), 176-194. Doi : https://doi.org/10.3917/rhmc.493.0176

Douyère, D. (2014). Communiquer la prière par l'image du corps : les Neuf manières de prier de saint Dominique Frédéric. Dans Fr. Lambert (dir.), Prières et propagandes. Études sur la prière dans les arènes publiques. Suivi du livre I de La Prière de Marcel Mauss (pp. 279-296). Paris : Hermann.

Douyère, D. (2018). Communiquer la doctrine catholique : textes et conversations durant le concile Vatican II d'après le journal d'Yves Congar. Genève : Labor et Fides.

Douyère, D. (2019). Youtube comme imaginaire de la relation et de la communication. « Coucou tout le monde! J'espère que vous allez bien ». Études digitales, 7(1), Youtoubeurs, youtubeuses : inventions subjectives, 215-239.

Durkheim, E. (1922). Éducation et sociologie. Paris, : Félix Alcan.

Duteil-Ogata et al. (2015). Le Religieux sur internet : textes et contextes. Paris : L'Harmattan.

Greiner, F. (2012). Fictions et dévotion au XVII siècle : une pédagogie de l'imaginaire. Littératures classiques, 79(3), 95-110. Doi : https://doi.org/10.3917/licla.079.0095

Hamman, A. G. (1952). Prières des premiers chrétiens. Paris : Fayard.

Helland, C. (2005). Online Religion as Lived Religion. Methodological Issues in the Study of Religious Participation on the Internet. Online-Heidelberg Journal of Religions on the Internet, 1(1), 1-16. Doi : https://doi.org/10.11588/heidok.00005823

Hernández, J.-P. (2020). Le discernement dans le cheminement spirituel. Lumen Vitae, 75(1), 61-68.

Hervieu-Léger, D. (1987). Faut-il définir la religion? Questions préalables à la construction d'une sociologie de la modernité religieuse. Archives de sciences sociales des religions, 63(1), 157-176. Doi : https://doi.org/10.3406/assr.1987.2418

Hoover, S. (2006), Religion in the Media Age. London-New York: Routledge.

Hoover, S. M. \& Clark, L. S. (2002). Practicing Religion in the Age of the Media: Explorations in Media, Religion, and Culture. New York: Columbia University Press.

Horvath, R. (2019). Évagre le Pontique, Chapitres sur la prière. Revue des sciences religieuses, 93(1-2), 187-188. Doi : https://doi.org/10.4000/rsr.6399

Jay, P. (1980). Saint Jérôme et le triple sens de l'Écriture. Revue d'Études Augustiniennes et Patristiques, 26(3-4), 214-227. 
Jonveaux, I. (2007). Une retraite de carême sur Internet. Archives de sciences sociales des religions, 139, 157-176. Doi : https://doi.org/10.4000/assr.9533

Lusted, D. (1986). Why Pedagogy? Screen, 27(5), 2-15. Doi : https://doi.org/10.1093/ screen/27.5.2

Martino, L. M. S. (2017). Mídia, religião e sociedade: das palavras às redes digitais. São Paulo : Paulus.

Mielle de Becdelièvre, D. (2003). La tradition de la lecture et la première bibliothèque cartusienne (fin $\mathrm{XI}^{\mathrm{e}}$ - début XII ${ }^{\mathrm{e}}$ siècle). Saint Bruno et sa postérité spirituelle. Dans J. Hoog, A. Girard \& D. Le Blévec (éds), Saint Bruno et sa postérité spirituelle. Actes du colloque international des 8 et 9 octobre 2001 à l'Institut catholique de Paris (pp. 219-229). Salzbourg, 2003.

Palazzo, É. (2016) Peindre, c'est prier. Anthropologie de la prière chrétienne. Paris : Cerf.

Palazzo, E. (2017). La liturgie dévotionnelle et les cinq sens : les neuf modes de prière de saint Dominique, Perspectives médiévales, 38, 1-15.

Penafria, M. (2009, April). Análise de Filmes-conceitos e metodologia(s). Dans VI Congresso Sopcom (Vol. 6, pp. 6-7). Lisboa : Universidade Lusófona. Disponible à : https://docplayer.com.br/265203-Analise-de-filmes-conceitos-e-metodologia-s.html

Pierron, J. P. (1995). La question du témoignage dans les Confessions de saint Augustin. Revue d'Études Augustiniennes et Patristiques, 41(2), 253-266. Disponible à : http://www.etudes-augustiniennes.paris-sorbonne.fr/IMG/pdf/AUGUST_1995_41_2_253.pdf

Robertson, D. (2011). Lectio Divina: The Medieval Experience of Reading. Collegeville: Liturgical Press.

Roques, R. (1960). H. de Lubac. Exégèse médiévale : les quatre sens de l'Écriture. Revue de l'histoire des religions, 158(2), 204-219.

Sevez, P. (2018). Pédagogie ignacienne. Études, 1, 69-80.

Siqueira de Santana, J. (2011). Entre bordados, cadernos e orações: a educação de meninas e as práticas educativas no orfanato de São Cristóvão e na escola da Imaculada Conceição (1922-1969) (Mémoire de master en Sciences de l'éducation). Universidade Federal de Sergipe, Brésil.

Trottmann, C. (2003). Contemplation et vie contemplative selon trois Chartreux : Guigues II, Hugues de Balma et Guigues du Pont : Quelques points de repère dans une évolution. Revue des sciences philosophiques et théologiques, 87(4), 633-680. Doi : https://doi.org/10.3917/rspt.874.0633

Vanoye, F. (2006). Ensaio sobre a análise fílmica (7ª́dição). Campinas : Papirus.

\section{Références audiovisuelles}

Brito, T. (2016). Como eu faço meu devocional | \#semi [Vidéo en ligne]. Repéré à https://www.youtube.com/watch?v=90IpxPq7ubU\&t=2s. Consulté 04.04.2019.

Keffer, F. (2017). Devocional diário - como eu faço meu devocional na pratica [Vidéo en ligne]. Repéré à https://www.youtube.com/watch?v=Gz5pVaQccM0. Consulté 12.04.2019. 
Néry, S. (2015) Como eu organizo meu caderno de oração | sete categorias [Vidéo en ligne]. Repéré à https://www.youtube.com/watch?v=TxXUtlI7DFE\&t=198s. Consulté 02.04.2019.

Ruiz, D. (2016). Devocional com Arte 6 - Meu caderno de devocionais [Vidéo en ligne]. Repéré à https://www.youtube.com/watch? $\mathrm{v}=\mathrm{C} 88$ PomifydE\&t=156s. Consulté 10.04.19.

Ruiz, D. (2016). Devocional com Arte 5. Como eu uso o meu caderno de devocionais - Devotional Journal [Vidéo en ligne]. Repéré à https://www.youtube.com/ watch? $=$ UqkSiwjyTWI\&t=1s. Consulté 05.04.2019.

Souza, M. (2015). O que é? E como fazer: Devocional By: Michelle Souza [Vidéo en ligne]. Repéré à https://www.youtube.com/watch?v=BMoul58kU0Q. Consulté le 02.04.2019.

Veríssimo, F. (2016). 5 motivos para começar um diário espiritual e (3 dicas de como fazê-lo!) [Vidéo en ligne]. Repéré à https://www.youtube.com/watch?v=wZm0oGpFqVE. Consulté le 25.05.2019. 


\section{(c) $(1) \Theta$}

BY NC ND Publié sous la licence Creative Common

«Attibution - pas d'utilisation Commerciale - Pas de Modification 4.0. International» (CC BY-NC-ND) 